\title{
Phylogenesis of prion protein
}

SIR - We have reconstructed the phylogeny for various mammal species based on the sequence of the prion protein gene. By tracing the pattern of amino-acid substitutions through the phylogeny, we have identified two pairs of derived substitutions uniquely shared by cattle (Bos taurus) and the hominoids. Although we have no specific explanation for these events, a phylogenetic analysis can help to indicate their significance. Two factors make the result compelling: the probability that it occurred by chance is estimated to be less than $1.2 \times 10^{-4}$; and the substitutions, although of a conservative nature, occur in a region of the gene postulated to be involved in the acquisition of prion diseases.

The prion protein is constitutively expressed in adult vertebrates and bound to the surface of neurons ${ }^{1}$. Its function is unknown, but in modified form it is associated with several neurodegen-

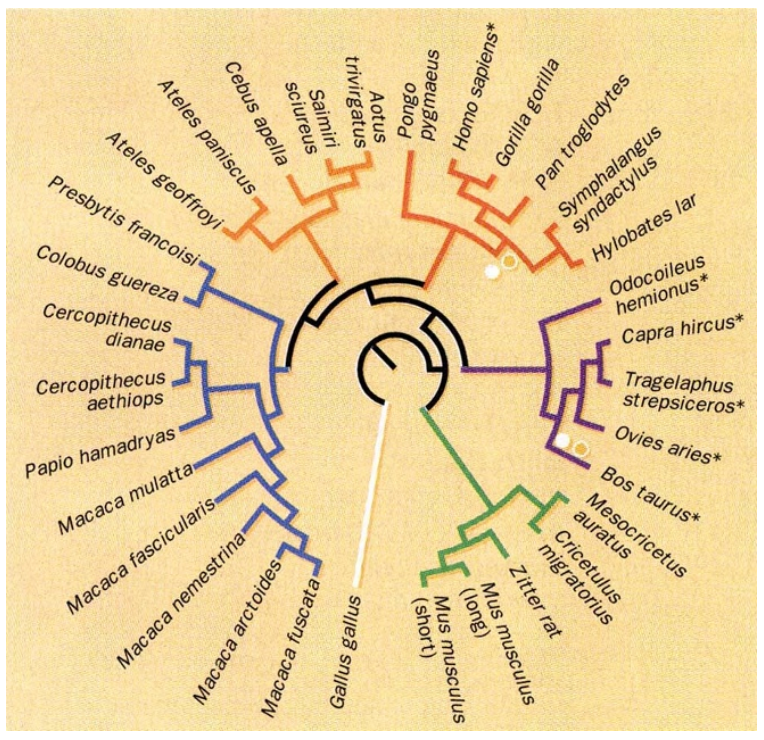

Phylogenetic relationships among the prion protein genes of 33 species of vertebrates estimated from a 750-nucleotide sequence using maximum-likelihood methods ${ }^{10}$. Higher taxa are colour-coded according to the following key: red (hominoids), purple (artiodactyls), green (rodents), blue (Old World monkeys), orange (New World monkeys), and aves in white used as an outgroup. The two reconstructed amino-acid substitutions are marked with circles. The white circle marks the Tyr-to-His (155) substitution, and the yellow circle the Asp-toSer (143) substitution. Of interest is that the gene tree topology is inconsistent with the species tree topology. For example, the Old World and New World monkeys are sister groups in the gene tree, whereas the Old World monkeys and the hominoids are sister taxa in the species tree. Homo is a sister group of Gorilla in the gene tree, whereas in the species tree, Homo is the sister species of Pan. Short- and long-latency mouse variants of a single species are also shown. The tree is a strict consensus of 100 DNAML maximum-likelihood trees, assuming a Kimura two-parameter model of evolution with a transition-transversion parameter of 2.0. Asterisks identify species known to contract spongiform encephalopathies naturally.
0.18 (95\% confidence interval $=0-0.55)$. Basing this calculation on the 11 sites that changed twice or more yields a figure of $0.0065(95 \%$ confidence interval $=$ $0-0.019)$. The event we observe is the only one of its kind in the phylogenetic tree.

Given the improbability that the convergence we describe occurred by chance, we can reflect on its biological significance. One possibility is that the substitutions confer resistance of the cellular prion protein to modification to the pathogenic form of the prion protein. Alternatively, they may improve the efficiency of this protein in the healthy tissue of these species. As an incidental consequence of the latter, these changes might also have predisposed humans towards a strain of prion disease occurring in $B$. taurus. Substitutions of a conservative biochemical nature have previously been implicated in disease symptoms in other studies of spongiform encephalopathies ${ }^{1}$. Mutations in the amino-acid residues between sites 90 and 145 have been shown to correlate with the onset of disease $^{7}$. Of the two substitutions we report, one falls within and the other is adjacent to this region.

The transmission of sheep-derived scrapie to humans has never been proved, despite the existence of endemic scrapie for more than 200 years ${ }^{8}$. The transmission of BSE to humans from cattle has recently been suggested ${ }^{9}$ to result in a previously unrecognized form of CJD with a novel neuropathology, although there is no direct evidence for this proposal. Although statistically minded scientists are ever mindful of Hume's dictum that correlation is not causation, we should nevertheless remain attentive to rare events that might be associated with the emergence of what may be a new strain of this disease.

David C. Krakauer

Mark Pagel

T. R. E. Southwood

Department of Zoology,

University of Oxford,

South Parks Road,

Oxford OX1 3PS, UK

Paolo M. de A. Zanotto

NERC Institute of Virology,

Mansfield Road,

Oxford OX1 3SR, UK

evolutionary amino-acid replacement in the phylogeny, seven show two, and four show three. If the 56 sites are taken as those showing any evidence of change, we obtain 1,540 possible different pairs of sites that might have shown the effect we observed. The expected number of correlated pairs of transitions such as we observed is thus $1,540 \times 1.2 \times 10^{-4}=$

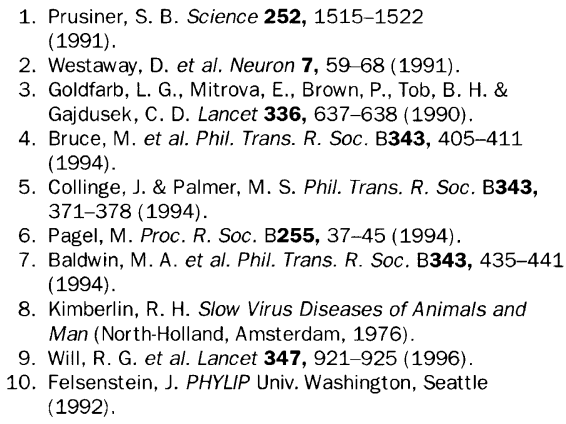

1. Prusiner, S. B. Science 252, 1515-1522 (1991).

2. Westaway, D. et al. Neuron 7, 59-68 (1991).

3. Goldfarb, L. G., Mitrova, E., Brown, P., Tob, B. H. \& Gajdusek, C. D. Lancet 336, 637-638 (1990)

4. Bruce, M. et al. Phil. Trans. R. Soc. B343, 405-411 (1994).

5. Collinge, J. \& Palmer, M. S. Phil. Trans. R. Soc. B343, 371-378 (1994).

6. Pagel, M. Proc. R. Soc. B255, 37-45 (1994)

7. Baldwin, M. A. et al. Phil. Trans. R. Soc. B343, 435-441 (1994).

8. Kimberlin, R. H. Slow Virus Diseases of Animals and Man (North-Holland, Amsterdam, 1976).

9. Will, R. G. et al. Lancet 347, 921-925 (1996)

10. Felsenstein, J. PHYLIP Univ. Washington, Seattle (1992). 